\title{
Déclenchement soudain de règles abondantes chez une femme de 34 ans
}

\author{
Roy Khalife MD, Lisa Duffett MD, Tzu-Fei Wang MD, Alan Tinmouth MD MSc
}

Citation : CMAJ 2021 August 3;193:E1173-6. doi : 10.1503/cmaj.210169-f

Voir la version anglaise de l'article ici : www.cmaj.ca/lookup/doi/10.1503/cmaj.210169

$\mathbf{U}$

ne femme de 34 ans a consulté aux urgences pour des règles abondantes depuis 1 journée. Elle a dit utiliser des serviettes hygiéniques épaisses ou même des couches et devoir les changer toutes les heures tellement ses saignements étaient abondants.

Un mois auparavant, elle avait connu une douleur pelvienne subite des suites d'une rupture de kyste ovarien hémorragique puis avait subi une kystectomie ovarienne par laparoscopie d'urgence qui s'est compliquée avec des pertes sanguines d'environ $900 \mathrm{~mL}$. Quatre jours après son congé postopératoire, elle a de nouveau consulté aux urgences en raison d'une aggravation de sa douleur abdominale et pelvienne. Son taux d'hémoglobine était alors de $41 \mathrm{~g} / \mathrm{L}$ (plage de référence 115155). Une seconde intervention d'urgence, par laparotomie ouverte, a été effectuée. Le saignement actif a finalement été arrêté par une salpingo-ovariectomie gauche. En période périopératoire, la patiente a reçu 4 culots globulaires, 4 unités de plasma congelé et $2 \mathrm{~g}$ d'acide tranexamique en 24 heures. Son taux d'hémoglobine était de $78 \mathrm{~g} / \mathrm{L}$ au moment du congé.

Avant ces visites à l'hôpital, elle n'avait jamais présenté de saignements anormaux ou de problèmes d'anémie, et son taux d'hémoglobine normal était de $120 \mathrm{~g} / \mathrm{L}$. Elle n'avait jamais eu de règles abondantes auparavant, ni d'épistaxis ou d'ecchymoses inhabituelles. Elle a eu un accouchement spontané par voie vaginale et subi des interventions dentaires sans complications hémorragiques. Elle n'avait pas d'antécédents familiaux de saignements anormaux et n'avait pris aucun anticoagulant ou anti-inflammatoire non stéroïdien. Son apport nutritionnel était adéquat et elle n'avait aucune restriction alimentaire.

À l'examen, la patiente paraissait anxieuse, son teint était pâle et elle était tachycarde. Elle présentait des saignements vaginaux actifs et de volumineuses ecchymoses aux membres inférieurs. Les analyses de laboratoires ont indiqué un taux d'hémoglobine de $52 \mathrm{~g} / \mathrm{L}$, avec un volume globulaire moyen, une numération plaquettaire et une numération leucocytaire normaux. Le frottis de sang périphérique a révélé une grave anémie, avec une quantité modérée de rouleaux érythrocytaires. Ses taux de ferritine et de vitamine B12 étaient à l'intérieur des limites de la normale. Un test de dépistage sérique de la bêtagonadotrophine chorionique humaine ( $\beta$-HCG) s'est révélé négatif, et l'échographie pelvienne était sans particularités.

\section{Parmi les analyses de laboratoire suivantes, laquelle serait inutile pour trouver l'origine des saignements chez cette patiente?}

a. Temps de prothrombine (prothrombin time, PT)/ratio international normalisé (RIN)

b. Temps de céphaline activée (activated partial thromboplastin time, aPTT)

c. Taux de fibrinogène

$\mathrm{d}$. Dosage des D-dimères

e. Temps de saignement

La réponse est « $\mathrm{e}$ ». Comme la patiente a des épisodes récurrents de saignements anormaux, elle devrait faire l'objet d'un dépistage de la diathèse hémorragique. Ses récents saignements soudains, de causes multiples (kyste ovarien hémorragique, hémorragie postopératoire, ménorragie subite et ecchymoses inhabituelles), font penser à une diathèse hémorragique acquise.

Les premières analyses à effectuer pour trouver l'origine de saignements anormaux sont la mesure du PT/RIN, du temps de céphaline activée et du taux de fibrinogène. On peut également recourir au dosage des $\mathrm{D}$-dimères pour déterminer la présence de coagulation intravasculaire disséminée. On n'a plus souvent recours au temps de saignement en raison d'une piètre standardisation, de la grande variabilité inter- et intra-examinateur et de la cicatrisation cutanée qu'il demande ${ }^{1}$.

L'anamnèse demeure le meilleur moyen de dépister une diathèse hémorragique. On portera une attention particulière aux foyers hémorragiques, à la gravité des saignements et à leur traitement, y compris des saignements survenant lors d'une intervention dentaire ou chirurgicale, des règles ou d'un accouchement. Les antécédents familiaux de saignements spontanés ou autres peuvent éveiller les soupçons quant à un trouble héréditaire de la coagulation. Même si différents médicaments sont associés à un risque hémorragique accru, les médecins devraient s'informer particulièrement de la prise d'antiplaquettaires (y compris d'antiinflammatoires non stéroïdiens) et d'anticoagulants.

Les analyses de laboratoire viennent compléter l'anamnèse complète. Dans des cas rares, les troubles rénaux, hépatiques et thyroïdiens exacerbent une prédisposition aux saignements et aux ecchymoses pour donner lieu à des règles abondantes chez les femmes préménopausées. Chez notre patiente, le PT/RIN, le 
Plasma de la patiente Plasma normal

(aPTT $\uparrow)$

(aPTT normal)

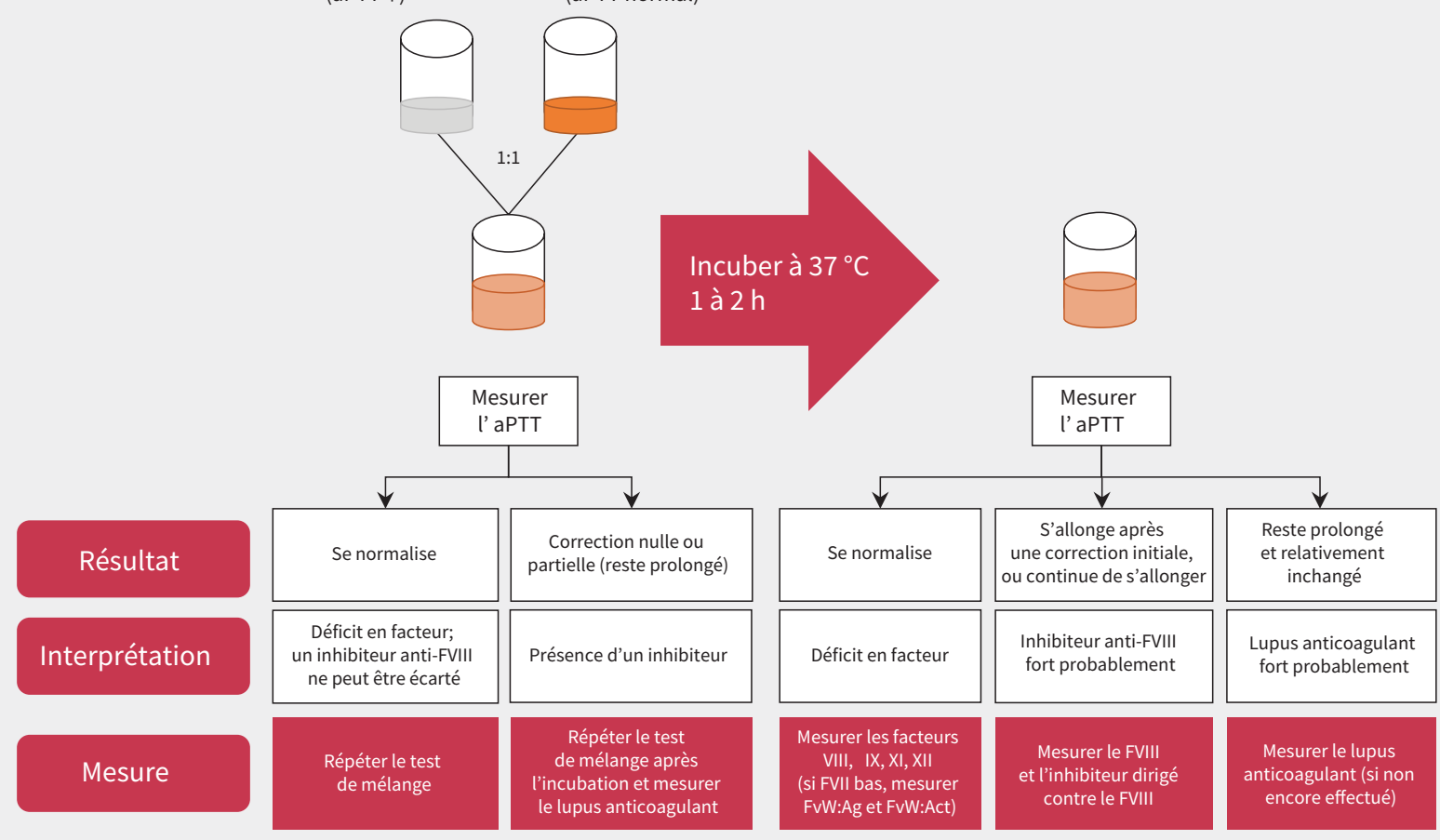

Figure 1 : Interprétation des tests de mélange et analyses de suivi suggérées ${ }^{1-3}$. Le plasma de la patiente, qui présente un temps de céphaline activée (aPTT) prolongé, est mélangé à du plasma normal selon un rapport de 1:1. Le temps de céphaline activée est mesuré immédiatement après le mélange et après 1-2 heures d'incubation de la solution à $37^{\circ} \mathrm{C}$. Si le temps de céphaline activée se situe dans les limites de la normale à l'analyse immédiate et à l'analyse postincubation, il faut soupçonner un déficit d'un facteur de coagulation, car le plasma normal vient ici compenser pour l'absence du ou des facteurs manquants. Si le temps de céphaline activée est entièrement ou partiellement corrigé au test immédiat, mais prolongé au test après incubation, il faut soupçonner la présence d'un inhibiteur dirigé contre le facteur VIII puisque la cinétique est visiblement dépendante du temps et de la température. La présence d'anticoagulants lupiques (des inhibiteurs non spécifiques) a tendance à prolonger le temps de céphaline activée et à ne pas faire voir de correction lors du mélange ou après 1 heure (aucun effet du temps ou de la température). Remarque: Act = activité, Ag = antigène, FvW = facteur de von Willebrand.

taux de fibrinogène et le dosage des D-dimères étaient normaux, rendant le diagnostic de coagulation intravasculaire disséminée peu probable. Par contre, le temps de céphaline activée était prolongé, à 46 secondes (plage de référence 19-28).

\section{Quel est le test à effectuer lorsque le temps de céphaline activée isolé est prolongé?}
a. Mesure du taux du facteur de von Willebrand
b. Épreuves de fonction plaquettaire
c. Temps de thrombine
d. Test de mélange (50:50)
e. Détection des anticoagulants lupiques

La réponse est « $d$ ». Un temps de céphaline activée isolé prolongé chez une patiente qui saigne sans avoir été exposée à une anticoagulothérapie devrait éveiller des soupçons quant à une anomalie de la voie intrinsèque de la coagulation (facteurs VIII, IX, XI), qui peut être causée par un déficit d'un facteur de coagulation ou l'interférence d'un inhibiteur sur l'activité des facteurs ${ }^{2,3}$. Même si le facteur XII fait aussi partie de la voie intrinsèque, sa carence donne lieu à une prolongation du temps de céphaline activée in vitro sans diathèse hémorragique asso- ciée $e^{2,4}$. Les anomalies dans le taux du facteur de von Willebrand ne prolongent pas le temps de céphaline activée, à moins d'un déficit concomitant en facteur VIII.

Le test de mélange (50:50), au cours duquel le plasma d'un patient est mélangé à du plasma normal dans un rapport de $1: 1^{2,3}$, s'effectue dans la plupart des laboratoires et sert à faire la distinction entre un déficit en facteur de coagulation et la présence d'inhibiteurs (figure 1$)^{4}$. C'est une épreuve que les médecins doivent demander, car les laboratoires ne l'effectuent pas d'emblée. Si un médecin soupçonne des taux de facteurs anormaux et que son centre est en mesure d'effectuer les analyses requises, il est préférable de faire les tests de mélange simultanément pour éviter de retarder le diagnostic ${ }^{4}$.

Nous avons rapidement procédé au test de mélange chez notre patiente. Deux heures plus tard, nous avons obtenu les résultats : son temps de céphaline activée était de 48,4 secondes et a été partiellement corrigé à 34,5 secondes par le mélange immédiat du plasma de la patiente avec du plasma normal provenant d'un groupe de donneurs (dont le temps de céphaline activée était de $25,4 \mathrm{~s}$ ). Après incubation de l'échantillon à $37^{\circ} \mathrm{C}$ pendant 1 heure, le temps de céphaline activée a rallongé, atteignant 57,6 secondes. 


\section{Quel diagnostic explique le plus probablement le tableau clinique et les résultats d'analyses chez cette patiente?}

a. Déficit congénital en facteur de coagulation

b. Inhibiteur acquis dirigé contre un facteur de coagulation

c. Maladie de von Willebrand

d. Syndrome de von Willebrand acquis

e. Présence d'anticoagulants lupiques

La réponse est « $\mathrm{b}$ ». Les résultats du test de mélange semblent indiquer la présence d'un inhibiteur dirigé contre un facteur de coagulation. La prolongation du temps de céphaline activée après incubation de l'échantillon à $37^{\circ} \mathrm{C}$ (ce qui démontre un effet du temps et de la température) ne s'observe en général que chez les patients porteurs d'un inhibiteur acquis dirigé contre le facteur VIII',3. Ce type d'inhibiteur, responsable de l'hémophilie A acquise, est le plus courant parmi les inhibiteurs acquis dirigés contre un facteur de coagulation spécifique ${ }^{4}$. En présence d'anticoagulants lupiques, le temps de céphaline activée pourrait être prolongé, mais il ne se corrigerait pas lors du test de mélange ${ }^{2}$. De plus, notre patiente avait des saignements importants, et la présence d'anticoagulants lupiques est plus souvent associée à des complications thrombotiques qu'à des complications hémorragiques².

Il faut donc soupçonner une hémophilie $A$ acquise chez tout patient en saignement qui présente un temps de céphaline activée isolé prolongé et les résultats au test de mélange décrits plus haut en plus de n'avoir aucun antécédent personnel ou familial de saignement. L'hémophilie $A$ acquise est confirmée par un taux de facteur VIII bas et la présence d'un inhibiteur dirigé contre ce même facteur, que des laboratoires d'hémostase spécialisés sont en mesure de quantifier ${ }^{4,5}$. Comme mesure complémentaire, nous avons aussi vérifié le taux du facteur de von Willebrand et son niveau d'activité, qui se trouvaient à l'intérieur des limites de la normale, permettant d'écarter un diagnostic de syndrome de von Willebrand acquis. Les analyses effectuées nous ont permis de poser un diagnostic d'hémophilie $\mathrm{A}$ acquise chez notre patiente.

\section{Parmi les options thérapeutiques suivantes, laquelle est contre-indiquée à cette étape-ci?}

a. Donner des soins de soutien et de réanimation, administrer de la solution physiologique salée et des culots globulaires

b. Administrer un traitement antifibrinolytique (p. ex., acide tranexamique)

c. Faire un tamponnement intra-utérin par ballonnet jusqu'à ce qu'une prise en charge définitive soit disponible

d. Consulter l'hématologue de garde d'un établissement ayant l'expertise clinique et de laboratoire nécessaire pour la prise en charge des troubles de la coagulation, en vue d'un traitement et d'un transfert immédiats

e. Consulter le gynécologue de garde au sujet de l'arrêt des règles et des traitements locaux possibles

La réponse est « $C$ ». Les patients soupçonnés de souffrir d'hémophilie A acquise devraient être transférés et pris en charge dans un centre spécialisé dans les troubles de la coagulation, leur diagnostic et leur traitement ${ }^{4}$. La prise en charge des saignements qui accompagnent l'hémophilie $A$ acquise repose sur l'administration de produits sanguins particuliers, dont des agents de contournement du facteur VIII ou des concentrés de facteur VIII porcin recombinant, qui ne sont pas disponibles dans tous les centres ${ }^{4}$. La desmopressine, les concentrés de facteur VIII humain recombinant et les perfusions de plasma sont généralement inefficaces et ne conviennent pas comme solutions de rechange ${ }^{4}$. L'administration d'acide tranexamique (un antifibrinolytique) et l'administration de préparations hormonales sont les options thérapeutiques de première intention en cas de saignements menstruels abondants aigus et sont également d'importants traitements d'appoint ${ }^{6}$. Le tamponnement intrautérin par ballonnet est une intervention effractive qui a peu de chances d'être efficace ou nécessaire parce que l'arrêt du saignement dépend du traitement de la coagulopathie sous-jacente ${ }^{6}$.

Nous avons administré des liquides intraveineux, des culots globulaires, de fortes doses d'œstrogènes et de l'acide tranexamique pour traiter les saignements menstruels abondants aigus et l'anémie marquée de notre patiente. Nous l'avons ensuite transférée dans un centre de traitement de l'hémophilie, et le diagnostic d'hémophilie $A$ acquise a été confirmé par un taux de facteur VIII bas de $0,33 \mathrm{UI} / \mathrm{mL}$ (plage de référence 0,50 1,50 ) et un titre détectable d'inhibiteur dirigé contre ce même facteur de 2,9 unités Bethesda/mL (plage de référence 0,0-0,5). Dans l'hémophilie A acquise, les taux de facteur VIII sont en général substantiellement réduits, passant sous la barre de $0,05 \mathrm{UI} / \mathrm{mL}(5 \%)$, mais des taux de 0,05 à $0,50 \mathrm{UI} / \mathrm{mL}$ ont aussi été associés à des épisodes de saignements ${ }^{7,8}$.

Notre patiente a présenté des saignements après des événements impliquant l'hémostase (p. ex., rupture de kystes ovariens, saignements postopératoires et règles), saignements qui sont prévisibles chez les patients qui ont un faible taux de facteur VIII. Le traitement œstrogénique à forte dose et l'acide tranexamique administrés ont mis fin à ses saignements menstruels. Un traitement immunosuppresseur à la prednisone, dosée à $1 \mathrm{mg} / \mathrm{kg} / \mathrm{j}$, a été entamé comme première tentative d'élimination de l'inhibiteur. Étant donné que de $30 \%$ à $50 \%$ des patients atteints d'hémophilie $A$ acquise présenteront une maladie maligne ou auto-immune sous-jacente ${ }^{5}$, des examens supplémentaires ont été effectués, notamment la recherche d'anticorps antinucléaires et de facteur rhumatoïde, l'électrophorèse des protéines et des épreuves d'imagerie pulmonaire, abdominale et pelvienne. Ces examens ont révélé un taux d'immunoglobuline monoclonale $\mathrm{G} K$ de $25 \mathrm{~g} / \mathrm{L}$. L'analyse de la moelle osseuse a confirmé le diagnostic de cancer des plasmocytes, mais les autres examens n'ont révélé aucune atteinte des organes cibles liée au myélome. L'hémophilie $A$ acquise de la patiente serait due au myélome multiple, pour lequel une chimiothérapie a été débutée.

\section{Discussion}

L'hémophilie A acquise est un trouble rare de la coagulation causé par la présence d'autoanticorps qui neutralisent le facteur VIII ${ }^{4,5}$. Son incidence est d'environ 1-1,5 cas par million, l'âge médian à l'acquisition est de 64 ans, et sa fréquence est la même chez les hommes et chez les femmes ${ }^{5,7}$. L'hémophilie A acquise est une urgence médicale. Elle doit être rapidement 
diagnostiquée et traitée, surtout en présence de saignements actifs. Ses signes distinctifs sont saignements modérés à graves (hématomes volumineux, saignements mucocutanés ou saignements des tissus mous) et ecchymoses ou saignements importants provenant de foyers inhabituels, en l'absence d'antécédents personnels ou familiaux de saignements ${ }^{5}$. Contrairement à l'hémophilie congénitale, les hémarthroses sont rares ${ }^{5,7}$. Les saignements ont tendance à être spontanés, mais peuvent aussi faire suite à un trauma ou une opération chirurgicale et être sans corrélation avec l'activité du facteur VIII 4,7,8. Non identifiée, I'hémophilie $A$ acquise peut mener à des hémorragies gravissimes, voire fatales ${ }^{7}$. On en découvre l'étiologie dans environ $50 \%$ des cas, les causes fréquentes étant notamment les cancers, les maladies auto-immunes, les infections et la prise de certains médicaments $5^{5,7}$. Elle peut aussi se manifester en période postpartum ${ }^{4,7}$.

La prise en charge de l'hémophilie $A$ acquise demande une expertise clinique et de laboratoire en hémostase et requiert généralement une consultation auprès d'un centre de traitement de l'hémophilie ${ }^{4}$. Les principales stratégies thérapeutiques comprennent l'arrêt des saignements actifs, l'élimination des inhibiteurs, et le traitement de la cause sous-jacente si elle a été découverte $^{4}$. Les agents hémostatiques utilisés pour mettre fin aux saignements actifs sont les agents de contournement du facteur VIII (p. ex., facteur VIIa recombinant [Niastase, NovoSeven], les concentrés de complexe prothrombique activé [p. ex., FEIBA]) et les concentrés de facteur VIII porcin recombinant (p. ex., Obizur). Étant donné qu'aucun essai clinique randomisé et contrôlé n'a comparé ces substances, le choix de l'agent hémostatique repose en général sur les caractéristiques des patients et des produits et sur l'expérience du médecin ${ }^{4}$. L'acide tranexamique devrait être utilisé avec circonspection chez les patients traités par facteur VIla recombinant ou concentré de complexe prothrombique activé, car leur utilisation concomitante a déjà causé des complications thromboemboliques ${ }^{4}$.

Les traitements immunosuppresseurs de première intention utilisés pour éliminer les inhibiteurs incluent la corticothérapie seule (pendant 4-6 semaines, suivie d'un sevrage graduel) et la corticothérapie assortie d'un cycle de cyclophosphamide (pendant 6 semaines) $)^{4}$. Dans la plus vaste étude de cohorte prospective multicentrique européenne sur l'hémophilie $A$ acquise, le traitement combiné a donné lieu à des taux de rémission plus élevés et à une rémission plus rapide, mais à un taux de mortalité global similaire par rapport à la corticothérapie seule, probablement en raison de la toxicité accrue et des complications infectieuses plus nombreuses liées au cyclophosphamide ${ }^{9}$. Le rituximab, administré 1 fois par semaine pendant un maximum de 4 cycles, peut aussi être utilisé en association avec la corticothérapie, mais est généralement envisagé en deuxième intention. Il donne des résultats similaires ou légèrement supérieurs à ceux de la corticothérapie seule 9 .

Le taux de mortalité hémorragique chez les patients atteints d'hémophilie A acquise, qui autrefois variait entre $20 \%$ et $30 \%$, a diminué dans les dernières années : il oscille désormais entre $3 \%$ (selon le registre le plus volumineux et plus ancien) ${ }^{7}$ et $9 \%$ (selon une étude de cohorte de 2 ans) ${ }^{10}$. Une récente étude rétrospective menée au Canada dans un seul centre indique que les patients atteints d'hémophilie A acquise ont un taux élevé d'hospitalisation $(87,5 \%)$ et d'admission aux soins intensifs $(12,5 \%)^{11}$. Les délais dans l'amorce du traitement sont associés à une baisse du taux de réponse et à des résultats cliniques moins bons ${ }^{4,8}$.

\section{Références}

1. Ma A, Mazepa M. Evaluation of the bleeding patient. In: Key NS, Makris M, Lillicrap D, editors. Practical Hemostasis and Thrombosis. 3rd ed. John Wiley \& Sons; 2017:63-78.

2. Hayward CPM, Moffat KA. Laboratory testing for bleeding disorders: strategic uses of high and low-yield tests. Int J Lab Hematol 2013;35:322-33.

3. Elbaz C, Sholzberg M. An illustrated review of bleeding assessment tools and common coagulation tests. Res Pract Thromb Haemost 2020;4:761-73.

4. Tiede A, Collins $P$, Knoebl $P$, et al. International recommendations on the diagnosis hemophilia and a treatment of acquired. Haematologica 2020;105:1791-801.

5. Tiede A, Zieger B, Lisman T. Acquired bleeding disorders. Haemophilia 2021;27(Suppl 3):5-13.

6. James AH, Kouides PA, Abdul-Kadir R, et al. Evaluation and management of acute menorrhagia in women with and without underlying bleeding disorders: consensus from an international expert panel. Eur J Obstet Gynecol Reprod Biol 2011;158:124-34.

7. Knoebl P, Marco P, Baudo F, et al. Demographic and clinical data in acquired hemophilia A: results from the European Acquired Haemophilia Registry (EACH2). J Thromb Haemost 2012;10:622-31.

8. Holstein K, Liu X, Smith A, et al. Bleeding and response to hemostatic therapy in acquired hemophilia A: results from the GTH-AH 01/2010 study. Blood 2020;136:279-87.

9. Collins P, Baudo F, Knoebl P, et al. Immunosuppression for acquired hemophilia A: results from the European Acquired Haemophilia Registry (EACH2). Blood 2012;120:47-55.

10. Collins PW, Hirsch S, Baglin TP, et al. Acquired hemophilia A in the United Kingdom: a 2-year national surveillance study by the United Kingdom Haemophilia Centre Doctors' organisation. Blood 2007;109:1870-7.

11. Jayakar JP, O'Neill N, Yan M, et al. Retrospective review of Acquired Haemophilia A from the largest Canadian Haemophilia treatment centre. Haemophilia 2018;24:e383-7.

Intérêts concurrents : Roy Khalife déclare avoir reçu des subventions de recherche de la Société canadienne de l'hémophilie, de Pfizer et du Département d'innovation en éducation médicale de l'Université d'Ottawa. Il a aussi reçu des honoraires de conférencier de Pfizer. Tzu-Fei Wang déclare avoir reçu une subvention de LEO Pharma et avoir été rémunérée pour sa participation aux comités consultatifs de Pfizer et de sServier. Aucun autre intérêt concurrent n'a été déclaré.

Cet article a été révisé par des pairs.

Les auteurs ont obtenu le consentement de la patiente.

Affiliations : Département de médecine de l'Université d'Ottawa, Hôpital d'Ottawa et Institut de recherche de l'Hôpital d'Ottawa, Ottawa, Ont.

Collaborateurs : Tous les auteurs ont contribué à la conception du travail, ont rédigé le manuscrit et en ont révisé de façon critique le contenu intellectuel important; ils ont donné leur approbation finale pour la version destinée à être publiée et assument l'entière responsabilité de tous les aspects du travail.

Propriété intellectuelle du contenu : Il s'agit d'un article en libre accès distribué conformément aux modalités de la licence Creative Commons Attributions (CC BY-NC-ND 4.0), qui permet l'utilisation, la diffusion et la reproduction dans tout médium à la condition que la publication originale soit adéquatement citée, que l'utilisation se fasse à des fins non commerciales (c.-à-d., recherche ou éducation) et qu'aucune modification ni adaptation n'y soit apportée. Voir : https://creativecommons.org/licenses/by-nc-nd/4.0/deed.fr.

Correspondance : Roy Khalife, rkhalife@toh.ca 